


\section{Die Mischna}

\section{Text, Übersetzung und ausführliche Erklärung \\ herausgegeben von den Univ.-Professoren \\ D. Dr. G. Beer-Heidelberg und D. o. Holtzmann-Gießen}

Alle Traktate der Mischna werden in demselben Format und in der Satzeinrichtung, wie der hier vorliegende Traktat, erscheinen.

Jeder Traktat erscheint für sich, sobald sein Bearbeiter das Ms. abgeschlossen hat; irgendwelche Reihenfolge kann also bei der Veröffentlichung der 63 Traktate nicht eingehalten werden.

Herausgeber und Verleger hoffen, die ganze Mischna im Verlaufe von 4-5 Jahren vorlegen zu können, da fast schon alle Traktate ihre Bearbeiter gefunden haben.

Alle zu demselben Seder gehörigen Traktate werden später auch zusammen in Buchform erhältlich sein, so daß die Mischna nach ihrem Abschluß in etwa 6 handlichen Bänden oder Doppelbänden zu haben sein soll.

Es kann entweder auf alle Traktate abonniert oder jeder Traktat einzeln erworben werden.

Der Verleger lädt zur Subskription auf diese Mischna-Ausgabe ein, indem er den Subskribenten einen bedeutend günstigeren Bezugspreis anbietet: der Druckbogen wird in der Subskription mit etwa 60 bis 70 Pfg., beim Einzelkauf mit etwa 70 bis $80 \mathrm{Pfg}$. berechnet werden.

Bei dem großen Umfang der Mischna wird der spätere Gesamtpreis für das vollständige Werk natürlich verhältnismäßig hoch sein. Dadurch aber, daß sich die Gesamtausgabe auf den Zeitraum von 4-5 Jahren erstreckt, hält sich der jährliche Aufwand für die Subskribenten in mäßigen Grenzen und steht jedenfalls in durchaus angemessenem Verhältnis zu der großen Wichtigkeit und dem hohen Wert des Gebotenen.

Bis jetzt sind folgende Traktate erschienen:

I. Seder: Zeraim, 1. Traktat: Berakot (Gebete), bearb. von Prof. D. Oscar Holtzmann-Gießen. (VIII u. 106 S.) M. 5.-, in der Subskr. M. 4.40

I. Seder: Zeraim, 2. Traktat: Pea (Vom Ackerwinkel), bearbeitet von Prof. Lic. Walter Bauer-Breslau. (V u. 74 S.) M. 4.-, in der Subskr. M. 3.50

I. Seder: Zeraim, 4. Traktat: Kil'ajim (Verbotene Mischgattungen), bearbeitet von Professor Dr. Karl Albrech t-Oldenburg. (VI und 87 Seiten.) M. 4.80

In der Subskription M. 4.20

I. Seder: Zeraim, 9. Traktat: Challa (Teighebe), bearb. von Prof. Dr. Karl Albrecht-Oldenburg. (IV u. 48 S.) M. 2.40, in der Subskr. M. 2.10

II. Seder: Moëd, 3. Traktat: Pesachim (Passahfest), bearb. von Prof. D. Dr. Georg Beer-Heidelberg. (XXIV u. 212 S.) M. 10.-, in der Subskr. M. 9.- 


\section{Die Mischna}

Text, Übersetzung und ausführliche Erklärung Mit eingehenden geschichtliehen und sprachlichen Einleitungen nnd textkritischen Anhängen

unter Mitwirkung von

Prof.Dr. Albrecht-Oldenburg/Prof. Lic. Bauer-Breslan/Prof.Lic. Dr. Benzinger-Toronto Oberlehrer Lic. Fiebig-Gotha / Pfarrer Lic. Dr. Frankenberg-Kassel / Prof. Lic. Dr. Frhr. v. Gall-Gießen/Prof.Dr. Ludw. Köhler-Zurich/Pfarrer Lic. Dr. Kramer-Gerichshain / Prof. D. Marti-Bern / Prof. D. Meinhold-Bonn / Prof. D. Dr. Nowack-Straßburg Prof. D. Dr. Rothstein-Münster i. W. / Past. Windfuhr-Hamburg herausgegeben von

Prof. D. Dr. G. Beer-Heidelberg und Prof. D. 0. Holtzmann-Gießen

I. Seder. Zeraim. 2. Traktat. Pea.

\section{Pea}

(Vom Ackerwinkel)

\section{Text, Übersetzung und Erklärung}

Nebst einem textkritischen Anhang

Von

Lic. Walter Bauer

a. o. Prof. d. Theologie an der Univ. Breslau

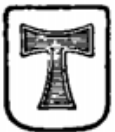

1914

Verlag von Alfred Töpelmann (vormals J. Ricker) in GieBen 
Alle Rechte vorbehalten

Copyright 1914 by Alfred Töpelmann

Druck von C. G. Röder G.m.b.H., Leipzig. 847214. 